\title{
One-dimension cubic-quintic Gross-Pitaevskii equation in Bose-Einstein condensates in a trap potential
}

\author{
C. Trallero-Giner ${ }^{1}$ and R. Cipolatti ${ }^{2}$ \\ ${ }^{1}$ Centro Brasileiro de Pesquisas Fisicas, Rua Xavier Sigaud 150, 22290-180 Rio de Janeiro-RJ, Brazil \\ ${ }^{2}$ Instituto de Matemática, Universidade Federal do Rio de Janeiro, C.P. 68530, Rio de Janeiro, RJ, Brasil
}

(Dated: October 25, 2019)

\begin{abstract}
By means of new general variational method we report a direct solution for the quintic self-focusing nonlinearity and cubic-quintic 1D Gross Pitaeskii equation (GPE) in a harmonic confined potential. We explore the influence of the $3 \mathrm{D}$ transversal motion generating a quintic nonlinear term on the ideal 1D pure cigar-like shape model for the attractive and repulsive atom-atom interaction in Bose Einstein condensates (BEC). Also, we offer a closed analytical expression for the evaluation of the error produced when solely the cubic nonlinear GPE is considered for the description of 1D BEC.
\end{abstract}

PACS numbers: 03.75.Lm, 03.75.Hh, 03.75.Kk, 05.45.Yv

\section{INTRODUCTION}

Nowadays one and quasi-one dimensional BoseEinstein condensates (BEC) are common experimental procedures ${ }^{1}$. The transition from $3 \mathrm{D}$ to $1 \mathrm{D}$ system was invoked long time $\mathrm{ago}^{2}$. In general, the $3 \mathrm{D}$ Gross-Pitaesvkii equation (GPE) cannot be factorized into transverse and longitudinal motions, nevertheless, under certain parameter regions we can assert that the BEC follows a 1D behavior (for a detailed discussion see Ref. 3). In the case of the harmonic trapping potential and considering that the atoms are tightly confined in two transverse directions, a transition to the quasi-1D description is possible. Starting with the standard 3D GPE, employing the adiabatic approximation and using the anzat wavefunction $\Psi(x, \mathbf{r} ; t)=\exp \left(-i \mu_{0} t / \hbar\right) \Phi(x) \chi(\mathbf{r} ; t)$, we can derive an effective 1D GPE, which describes the physical characteristics of the cigar-like shape condensate $\underline{\underline{4}-\underline{6}}$

$$
-\frac{\hbar^{2}}{2 m} \frac{d^{2} \Phi}{d x^{2}}+\frac{1}{2} m \omega^{2} x^{2} \Phi+g_{1 D}|\Phi|^{2} \Phi-g|\Phi|^{4} \Phi=\mu_{0} \Phi,
$$

where $\mu_{0} \in \mathbb{R}$ is the chemical potential, $m$ is the atomic mass, $\omega$ is the longitudinal harmonic oscillator frequencies, and $g_{1 D} \in \mathbb{R}, g \in \mathbb{R}$ are the effective $1 \mathrm{D}$ nonlinear self-interaction coefficients. These two coefficients depend on the total number $N$ of particles in the condensate, the transverse harmonic oscillator frequency $\omega_{\mathbf{r}}$ and the scattering length $a_{s}\left(a_{s}>0\right.$ or $a_{s}<0$ for attractive or repulsive interatomic interaction, respectively) by the relations $g_{1 D}=2 a_{s} N \hbar \omega_{\mathrm{r}}$ and $g=6 \ln (4 / 3) g_{1 D}^{2} / \hbar \omega_{\mathrm{r}^{\mathrm{r}}}$, where we have chosen for the stationary state $\Phi$ the normalization condition $\int_{\mathbb{R}} d x|\Phi|^{2}=1$.

Equation (1) is a cubic-quintic nonlinear Schödinger equation (NLSE) with real coefficients. The presence of the $-g|\Phi|^{4}$ term in (11) is due to the deviation from one dimension on the longitudinal condensate dynamics, i.e. a residual three dimensionality on an effective onedimensional GPE. In the case of a homogeneous medium, i.e. assuming that $\omega$ is zero, the cubic-quintic NLSE was widely used to describe the physical process of an optical medium with a nonlinear polarization including susceptibilities up to fifth order ${ }^{7}$. Also, the soliton solutions have been extensively studies in Refs. 5, 6, 8.

As we have mentioned above, Eq. (11) is the cigar-like shape approach from the 3D GPE. An important issue is the range of validity of (1), which is directly linked to the existence and stability of set of ground states solutions of the 3D NLSE. It is well known that for any value of $a_{s}>0$ the 3D GPE does not collapse ${ }^{4}$. However, for attractive interatomic interactions, the solution is dynamically stable if and only if $a_{s}$ is within the range $\frac{6.9}{.9}$

$$
\frac{N\left|a_{s}\right|}{a_{\perp}}<0.627
$$

with $a_{\perp}=\sqrt{\hbar / m \omega_{\mathbf{r}}}$. Hence, the validity of the cigar-like shape approach represented by Eq. (11) is also restricted to the constrain (2).

Rescaling to dimensionless variables $l_{o}=\sqrt{\hbar / m \omega}$, $\xi=x / l_{o}, \lambda=2 g_{1 D} /\left(l_{o} \hbar \omega\right), \mu=2 \mu_{0} /(\hbar \omega), \psi(\xi)=$ $\Phi\left(\xi l_{o}\right) / \sqrt{l_{o}}, \varepsilon=3 \ln (4 / 3) \omega / \omega_{\mathbf{r}}$, Eq. (1) can be cast as

$$
-\frac{d^{2} \psi}{d \xi^{2}}+\xi^{2} \psi+\lambda|\psi|^{2} \psi-\varepsilon \lambda^{2}|\psi|^{4} \psi=\mu \psi .
$$

The main task of this paper is the implementation of a more general variational mathematical approach to solve Eq. (3). Based on this result, we provide approximate solutions for the order parameter, the chemical potential and minimal energy for the quintic and cubic-quintic GPEs. The paper is organized as follows. First, in Sec. II we present the bases of our formalism, i.e., we present exact formulae for the energy and the chemical potential as functions of relevant parameters of (3). By considering a trial function for the ground state, we derive in Sec. III a representation for the energy, $E_{a p p}(\lambda)$, and chemical potential, $\mu_{a p p}(\lambda)$. Section IV is devoted to the application of our results to get explicit approximate solutions for the quintic and also cubic-quintic NLSEs. An estimation of the error due to the influence of the interaction 
between the axial and radial degrees of freedom on the 1D cigar-shape model is presented both graphically and analytically as function of the self-interaction parameter $\lambda$ and the coefficient $\varepsilon$ leading the quintic nonlinear term.

\section{CUBIC-QUINTIC NONLINEAR GROSS-PITAEVSKII EQUATION}

In the following we will consider a more general nonlinear Gross-Pitaevskii equation

$$
-\frac{d^{2} \psi}{d \xi^{2}}+\xi^{2} \psi+a \lambda|\psi|^{2} \psi-b \lambda^{2}|\psi|^{4} \psi=\mu \psi, \quad \xi \in \mathbb{R},
$$

where $a \geq 0$ and $b$ are real constants.

$$
\text { Let } \mathbf{V}=\left\{\left.\psi \in H^{1}(\mathbb{R})\left|\int_{\mathbb{R}} \xi^{2}\right| \psi(\xi)\right|^{2} d \xi<+\infty\right\} \text { be the }
$$

Hilbert space endowed $\underline{10}$ with the norm

$$
\|\psi\|_{\mathbf{V}}=\left[\int_{\mathbb{R}}|\psi(\xi)|^{2} d \xi+\int_{\mathbb{R}} \xi^{2}|\psi(\xi)|^{2} d \xi\right]^{1 / 2}
$$

and the corresponding inner product

$$
(\phi \mid \psi)_{\mathbf{V}}=\int_{\mathbb{R}}\left(\frac{d \phi}{d \xi} \frac{d \psi}{d \xi}+\xi^{2} \phi(\xi) \psi(\xi)\right) d \xi .
$$

In $\mathbf{V}$ we define the energy functional

$$
\begin{aligned}
E_{\lambda}[\psi]= & \int_{\mathbb{R}}\left|\frac{d}{d \xi} \psi(\xi)\right|^{2} d \xi+\int_{\mathbb{R}} \xi^{2}|\psi(\xi)|^{2} d \xi \\
& +\frac{a \lambda}{2} \int_{\mathbb{R}}|\psi(\xi)|^{4} d \xi-\frac{b \lambda^{2}}{3} \int_{\mathbb{R}}|\psi(\xi)|^{6} d \xi
\end{aligned}
$$

We denote by $\mathcal{G}_{\lambda}$ the set of ground states of Eq. (4), i.e., the set of functions of $\mathbf{V}$ that minimize the energy functional $E_{\lambda}[\psi]$ under the condition

$$
Q[\psi]=\int_{\mathbb{R}}|\psi(\xi)|^{2} d \xi=1 .
$$

Notice that in the case of attractive interaction where $b>0$, it is possible to show (by applying the GagliardoNirenberg inequalities) that the set of ground states $\mathcal{G}_{\lambda}$ is nonempty if the condition $|\lambda|<2 / \sqrt{|b|}$ is satisfied. Hence, for $\psi_{\lambda} \in \mathcal{G}_{\lambda}$ we obtain

$$
\frac{d E_{\lambda}\left[\psi_{\lambda}\right]}{d \lambda}=\left\langle\frac{\delta E_{\lambda}\left[\psi_{\lambda}\right]}{\delta \psi_{\lambda}} ; \frac{d \psi_{\lambda}}{d \lambda}\right\rangle+\frac{a}{2}\left\|\psi_{\lambda}\right\|_{4}^{4}-\frac{2 b \lambda}{3}\left\|\psi_{\lambda}\right\|_{6}^{6},
$$

where

$$
\|\psi(\xi)\|_{4}^{4}=\int_{\mathbb{R}}|\psi(\xi)|^{4} d \xi \text { and }\|\psi(\xi)\|_{6}^{6}=\int_{\mathbb{R}}|\psi(\xi)|^{6} d \xi
$$

are the usual norms of the standard Banach spaces $L^{4}(\mathbb{R})$ and $L^{6}(\mathbb{R})$. Taking into account the relation between the energy and the chemical potential, i.e. $\delta E_{\lambda}\left[\psi_{\lambda}\right] / \delta \psi_{\lambda}=$ $\mu \psi_{\lambda}$, it follows that

$$
\begin{aligned}
\left\langle\frac{\delta E_{\lambda}\left[\psi_{\lambda}\right]}{\delta \psi_{\lambda}} ; \frac{d \psi_{\lambda}}{d \lambda}\right\rangle & =\mu\left\langle\psi_{\lambda} ; \frac{d \psi_{\lambda}}{d \lambda}\right\rangle \\
& =\frac{\mu}{2} \frac{\delta Q_{\lambda}\left[\psi_{\lambda}\right]}{\delta \psi_{\lambda}}=0 .
\end{aligned}
$$

Hence, from Eqs. (7) and (9) we obtain the useful formula

$$
\frac{d E_{\lambda}\left[\psi_{\lambda}\right]}{d \lambda}=\frac{a}{2}\left\|\psi_{\lambda}\right\|_{4}^{4}-\frac{2 b \lambda}{3}\left\|\psi_{\lambda}\right\|_{6}^{6} .
$$

Thus, the minimum energy is given by

$$
E_{\min }(\lambda)=1+\frac{a}{2} \int_{0}^{\lambda}\left\|\psi_{s}\right\|_{4}^{4} d s-\frac{2 b}{3} \int_{0}^{\lambda} s\left\|\psi_{s}\right\|_{6}^{6} d s .
$$

Moreover, from Eq. (3) is straightforward that the chemical potential can be written as

$$
\mu_{\min }(\lambda)=E_{\min }(\lambda)+\frac{a \lambda}{2}\left\|\psi_{\lambda}\right\|_{4}^{4}-\frac{2 b \lambda^{2}}{3}\left\|\psi_{\lambda}\right\|_{6}^{6} .
$$

or equivalently 10

$$
\begin{aligned}
\mu_{\min }(\lambda)= & 1+\frac{a}{2}\left[\lambda\left\|\psi_{\lambda}\right\|_{4}^{4}+\int_{\mathbf{0}}^{\lambda}\left\|\psi_{s}\right\|_{4}^{4} d s\right] \\
& -\frac{2 b}{3}\left[\lambda^{2}\left\|\psi_{\lambda}\right\|_{6}^{6}+\int_{\mathbf{0}}^{\lambda} s\left\|\psi_{s}\right\|_{6}^{6} d s\right] .
\end{aligned}
$$

It is important to remark that Eqs. (10) and (11) are exact under the condition of knowing the ground state $\psi_{\lambda} \in \mathcal{G}_{\lambda}$ and therefore, independent of the method or approach we employe to get the solution of the Eq. (3).

\section{APPROXIMATE FORMULAE}

It is possible to show $\underline{\underline{10}}$ that any solution of Eq. (4) belonging to the Hilbert space $\mathbf{V}$ has the asymptotic behavior $\exp \left(-\tau \xi^{2}\right)$, with $\tau>0$, as $|\xi| \rightarrow \infty$. So, to evaluate the minimal energy $E_{\min }(\lambda)$, we can consider for the ground state the trial function 


$$
\psi_{\tau}(\xi)=\left(\frac{2 \tau}{\pi}\right)^{1 / 4} \exp \left(-\tau \xi^{2}\right)
$$

Using the function (12) and evaluating the energy functional (5), we obtain the algebraic expression

$$
E_{\lambda}\left[\psi_{\tau}\right]=\left(1-\epsilon \lambda^{2}\right) \sigma^{2}+\frac{1}{4 \sigma^{2}}+\frac{a \lambda}{2 \sqrt{\pi}} \sigma
$$

with $\epsilon=2 b /(3 \sqrt{3} \pi)$ and $\sigma=\sqrt{\tau}$. In the case $\epsilon \leq 0$ the Eq. (13) presents a global minimum in $(0,+\infty)$ for any $a$ and $\lambda \in \mathbb{R}$, but for $\epsilon>0$ a global minimum is guaranteed if $|\lambda|<1 / \sqrt{\epsilon}=\sqrt{3 \pi \sqrt{3}} / \sqrt{2 b}$.

Let $\sigma(\lambda)=\sqrt{\tau(\lambda)}$ be the minimizer of Eq. (13), in this way the ground state solution in $\mathcal{G}_{\lambda}$ can be searched considering the function $\varphi_{\lambda}(\xi):=\psi_{\tau(\lambda)}(\xi)$. By using Eqs. (10) and (11) we obtain the approximate energy, $E_{a p p}(\lambda)$, and chemical potential, $\mu_{a p p}(\lambda)$, namely

$$
E_{a p p}(\lambda)=1+\frac{a}{2 \sqrt{\pi}} \int_{\mathbf{0}}^{\lambda} \sigma(s) d s-2 \epsilon \int_{\mathbf{0}}^{\lambda} s \sigma^{2}(s) d s
$$

and

$$
\begin{aligned}
\mu_{a p p}(\lambda)=1+\frac{a}{2 \sqrt{\pi}}\left[\lambda \sigma(\lambda)+\int_{\mathbf{0}}^{\lambda} \sigma(s) d s\right] \\
-2 \epsilon\left[\lambda^{2} \sigma^{2}(\lambda)+\int_{\mathbf{0}}^{\lambda} s \sigma^{2}(s) d s\right] .
\end{aligned}
$$

It becomes clear that $|\lambda|<2 / \sqrt{b}<\lambda_{S}=\sqrt{3 \pi \sqrt{3}} / \sqrt{2 b}$, ensuring that the set $\mathcal{G}_{\lambda}$ is nonempty and that the functions $E_{a p p}(\lambda)$ and $\mu_{a p p}(\lambda)$ can be good approximations of $E_{\min }(\lambda)$ and $\mu_{\min }(\lambda)$, respectively.

\section{APPLICATIONS AND DISCUSSION OF THE RESULTS}

Explicit formulae (10), (11) and the approximate relationships (14), (15) are among the main results of our work. Nevertheless, more explicit expressions for the order parameter $\varphi_{\lambda}$, the energy and the chemical potential as a function of the atom-atom interaction term would be desirable. To do so, let $\sigma_{\min }=\sigma_{\min }(a, \epsilon ; \lambda)$ be the global minimizer of Eq. (13). Thus, Eqs. (14) and (15) with $\sigma=\sigma_{\min }$ allow to obtain the dependence $E_{a p p}$ and $\mu_{a p p}$ on the relevant physical parameters $a, \epsilon$ and $\lambda$. Depending on the values of $\lambda$ and the sign of the parameter $\epsilon$, we can distinguish several phases linked to both type of interaction strengths, i.e. i) pure attractive $(\lambda<0, \epsilon>0)$, ii) pure repulsive $(\lambda>0, \epsilon<0)$, and iii) a mixture $(\lambda<0, \epsilon<0$ or $\lambda>0, \epsilon<0)$.
More precisely, the value of $\tau_{\min }\left(\sigma_{\min }(\lambda):=\sqrt{\tau_{\min }}\right)$ that minimizes the function $\tau \longmapsto E_{\lambda}\left[\psi_{\tau}\right]$ fulfil the equation

$$
\left(1-\epsilon \lambda^{2}\right) \sigma^{4}+\frac{a \lambda}{4 \sqrt{\pi}} \sigma^{3}=\frac{1}{4} .
$$

Notice that, for $\epsilon \leq 0$ or $\epsilon>0$ under the condition $|\lambda|$ $<1 / \sqrt{\epsilon}$, the function (13) is strictly convex and coercive on the interval $(0,+\infty)$, and in consequence Eq. (16) has a unique solution, while for $|\lambda|>1 / \sqrt{\epsilon}$ the function (13) is not bounded from below.

\section{A. Quintic NLSE}

Firstly and for sake of comparison, we will consider the quintic NLSE in a harmonic potential ${ }^{11}$. Choosing $a=0$, from (16) we have

$$
\sigma_{\varphi^{5}}^{2}=\frac{1}{2\left(1-\epsilon \lambda^{2}\right)^{1 / 2}} .
$$

Inserting (17) into Eqs. (14) and (15) with $a=0$, we

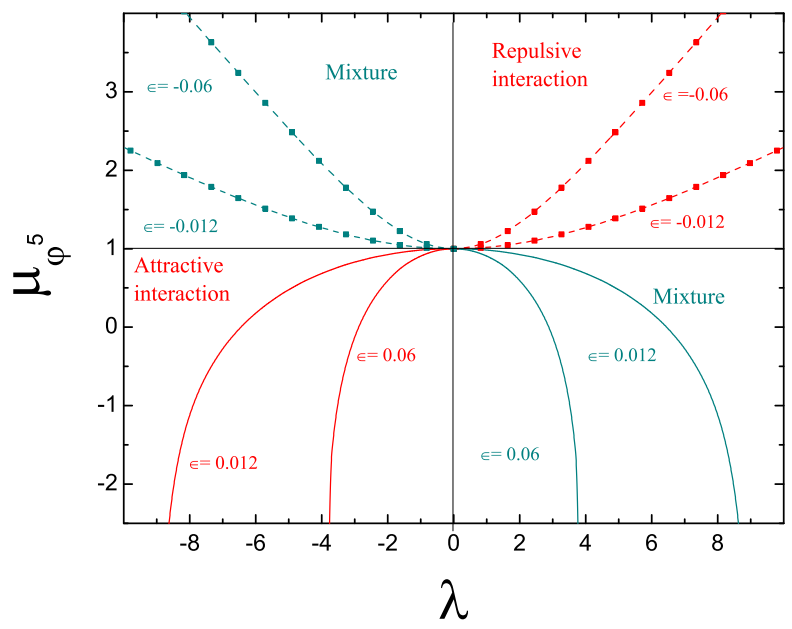

FIG. 1: (Color online) $\mu_{\varphi^{5}}-\lambda$ map for the parameter values $\epsilon= \pm 0.06$ and \pm 0.012 . According to the signs of $\lambda$ and $\epsilon$ the character of the $\mu_{\varphi^{5}}$, as approximated solution of Eq. (4) with $a=0$, can be mapped into fourth zones: $\lambda>0, \epsilon<0$ -pure repulsive interaction; $\lambda<0, \epsilon>0$-pure attractive interaction; $\lambda>0, \epsilon>0$ and $\lambda<0, \epsilon<0$-mixture region.

obtain for the approximate energy $E_{\varphi^{5}}$ and chemical potential $\mu_{\varphi^{5}}$ the expressions

$$
E_{\varphi^{5}}=\left(1-\epsilon \lambda^{2}\right)^{1 / 2}
$$

and

$$
\mu_{\varphi^{5}}=\left(1-\epsilon \lambda^{2}\right)^{1 / 2}-\frac{\epsilon \lambda^{2}}{\left(1-\epsilon \lambda^{2}\right)^{1 / 2}}
$$


Figure 1 displays the $\mu_{\varphi^{5}}-\lambda$ map diagram for several values of the parameter $\epsilon$. It can be seen that the reduced chemical potential $\mu_{\varphi^{5}}=2 \mu_{0} /(\hbar \omega)$ shows a strong dispersion as a function of $\lambda$, moreover and following the symmetry properties of Eq. (19), we observe that $\mu_{\varphi^{5}}$ increases (decreases) for the pure repulsive phase, $\lambda>0$, $\epsilon<0$ (pure attractive phase, $\lambda<0, \epsilon>0$ ), while in the mixture region an opposite behavior is reached with respect to the pair of values $(\lambda, \epsilon)$.

Following Eqs. (12) and (17) we obtain for the wavefunction $\varphi_{\varphi^{5}, \lambda}$ the expression

$$
\varphi_{\varphi^{5}, \lambda}(\xi)=\frac{1}{\left[\pi\left(1-\epsilon \lambda^{2}\right)^{1 / 2}\right]^{1 / 4}} \exp \left[-\frac{\xi^{2}}{2\left(1-\epsilon \lambda^{2}\right)^{1 / 2}}\right]
$$

valid for $1>\lambda^{2} \epsilon$. The above obtained wave function exhibits different behavior depending on the sign of $\epsilon$ and independent of the type interaction (attractive with $\lambda<0$ or repulsive for $\lambda>0$ ). The function becomes effectively less confined for $\epsilon<0$, i.e. $\varphi_{\varphi^{5}, \lambda}$ is delocalized and its maximum decreases, while for $\epsilon>0$ the function $\varphi_{\varphi^{5}, \lambda}(\xi)$ gets more localized and the maximum increases as the nonlinear potential $\lambda^{2} \epsilon$ increases.

\section{B. Cubic-quintic NLSE}

Although one can solve Eq. (16) numerically and to obtain from Eqs. (14) and (15) the energy $E_{\varphi^{3}-\varphi^{5}}(\lambda, b)$ and the chemical potential $\mu_{\varphi^{2}-\varphi^{5}}(\lambda, b)$, it will be very useful to report explicit compact approximate solution of the cubic-quintic nonlinear 1D GPE (4). Searching the solution of Eq. (16) with $a=1$ and $b=\varepsilon>0$ as a Taylor series on $\lambda, \sigma_{\varphi^{3}-\varphi^{5}}=\sum_{n=0}^{\infty} \sigma_{n} \lambda^{n}$, we get

$$
\begin{aligned}
\sigma_{\varphi^{3}-\varphi^{5}}= & \frac{1}{\sqrt{2}}-\frac{\lambda}{16 \sqrt{\pi}}+ \\
& \frac{\sqrt{2}}{2 \pi}\left(\frac{3}{256}+\frac{\varepsilon}{6 \sqrt{3}}\right) \lambda^{2}- \\
& \frac{1}{2 \pi \sqrt{\pi}}\left(\frac{1}{512}+\frac{\varepsilon}{12 \sqrt{3}}\right) \lambda^{3}+ \\
& \frac{\sqrt{2}}{3 \pi^{2} 2^{11}}\left(\frac{45}{128}+\frac{42 \varepsilon}{\sqrt{3}}\right) \lambda^{4}+\ldots
\end{aligned}
$$

Under the condition $|\varepsilon|<<1$ and by substituting Eq. (21) in (14) and (15) we have, for $\lambda$ small enough

$$
\begin{aligned}
& E_{\varphi^{3}-\varphi^{5}}=E_{\varphi^{3}}(\lambda)+\Delta E_{\varphi^{3}-\varphi^{5}}(\lambda, \varepsilon), \\
& \mu_{\varphi^{3}-\varphi^{5}}=\mu_{\varphi^{3}}(\lambda)+\Delta \mu_{\varphi^{3}-\varphi^{5}}(\lambda, \varepsilon),
\end{aligned}
$$

where $E_{\varphi^{3}}(\lambda)$ and $\mu_{\varphi^{3}}(\lambda)$ correspond to the energy and the chemical potential, respectively, for the cubic NLSE and are given, up to the 5 th order, by 10

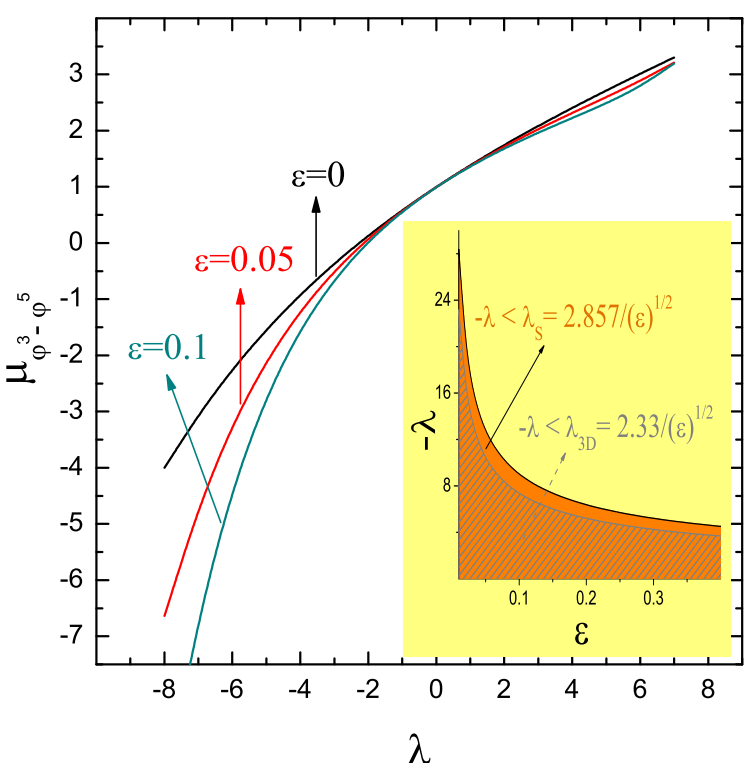

FIG. 2: (Color online) Reduced chemical potential $\mu_{\varphi^{3}-\varphi^{5}}$ as a function of the dimensionless self-interaction parameter $\lambda$, calculated from Eqs. (22)-(27) and several values of coefficient $\varepsilon$. Inset: $\lambda-\varepsilon$ map showing the validity ranges of Eqs. (21)(27) and the stability region of 3D GPE given by $-\lambda<\lambda_{S}=$ $2.857 /(\varepsilon)^{1 / 2}$ (orange) and $-\lambda<\lambda_{3 D}=2.33 /(\varepsilon)^{1 / 2}$ (gray lines), respectively.

$$
\begin{aligned}
E_{\varphi^{3}}(\lambda)= & 1+\frac{\sqrt{2} \lambda}{4 \sqrt{\pi}}-\frac{1}{\pi} \frac{1}{64} \lambda^{2}+\frac{\sqrt{2}}{12 \pi \sqrt{\pi}} \frac{3}{256} \lambda^{3} \\
& -\frac{1}{32 \pi^{2}} \frac{1}{256} \lambda^{4}+\frac{\sqrt{2}}{2^{13} \pi^{2} \sqrt{\pi}} \frac{3}{2^{6}} \lambda^{5}, \\
\mu_{\varphi^{3}}(\lambda)= & 1+\frac{\sqrt{2} \lambda}{2 \sqrt{\pi}}-\frac{1}{\pi} \frac{3}{64} \lambda^{2}+\frac{\sqrt{2}}{\pi \sqrt{\pi}} \frac{1}{256} \lambda^{3} \\
& -\frac{5}{32 \pi^{2}} \frac{1}{256} \lambda^{4}+\frac{3 \sqrt{2}}{2^{12} \pi^{2} \sqrt{\pi}} \frac{3}{2^{6}} \lambda^{5} .
\end{aligned}
$$

The terms $\Delta E_{\varphi^{3}-\varphi^{5}}(\lambda, \varepsilon)$ and $\Delta \mu_{\varphi^{3}-\varphi^{5}}(\lambda, \varepsilon)$ tell us how much error is made by neglecting the potential $-\varepsilon \lambda^{2}|\psi|^{4}$ in the $1 \mathrm{D}$ cigar-like shape model. These quantities are expressed by

$$
\begin{aligned}
\Delta E_{\varphi^{3}-\varphi^{5}}(\lambda, \varepsilon)= & \frac{\sqrt{3}}{9 \pi}\left[-\lambda+\frac{\sqrt{2}}{8 \sqrt{\pi}} \lambda^{2}\right. \\
& \left.-\frac{1}{32 \pi} \lambda^{3}+\frac{7 \sqrt{2}}{2^{11} \pi \sqrt{\pi}} \lambda^{4}\right] \varepsilon \lambda,
\end{aligned}
$$

and 


$$
\begin{aligned}
\Delta \mu_{\varphi^{3}-\varphi^{5}}(\lambda, \varepsilon)= & \frac{\sqrt{3}}{3 \pi}\left[-\lambda+\frac{\sqrt{2}}{6 \sqrt{\pi}} \lambda^{2}-\right. \\
& \left.\frac{5}{96 \pi} \lambda^{3}+\frac{7 \sqrt{2}}{2^{10} \pi \sqrt{\pi}} \lambda^{4}\right] \varepsilon \lambda .
\end{aligned}
$$

Figure (2) is devoted to the calculated chemical potential $\mu_{\varphi^{3}-\varphi^{5}}$ using Eqs. (23), (25), and (27) as a function of $\lambda$ for $\varepsilon=0,0.05$, and 0.1. First, by comparing Figs. (1) and (2) we see the strong qualitative difference between the two types equations here considered, quintic and cubicquintic NLSEs. Notice that this difference is remarkable even at $\lambda \approx 0$. From the Fig. (2) we can assert that the cigar-like shape approximation retaining term up to $|\phi|^{2}$ is a good approach solely for the repulsive case. The chemical potential (and also, the energy) for $\lambda>0$ and $\omega<<\omega_{\mathbf{r}}$ is almost independent of $\varepsilon=3 \ln (4 / 3) \omega / \omega_{\mathbf{r}}$, while a not negligible contribution is reached to $\mu_{\varphi^{3}-\varphi^{5}}$ if the atom-atom interaction is attractive, even for very small value of $\varepsilon$. In the former case the term $-\varepsilon \lambda^{2}|\phi|^{4}$ is responsible for the strong obtained dispersion compared to the $\varepsilon=0$ limit. In order to understand from the physical point of view the behavior of the chemical potential on $\lambda$, we define the effective potential

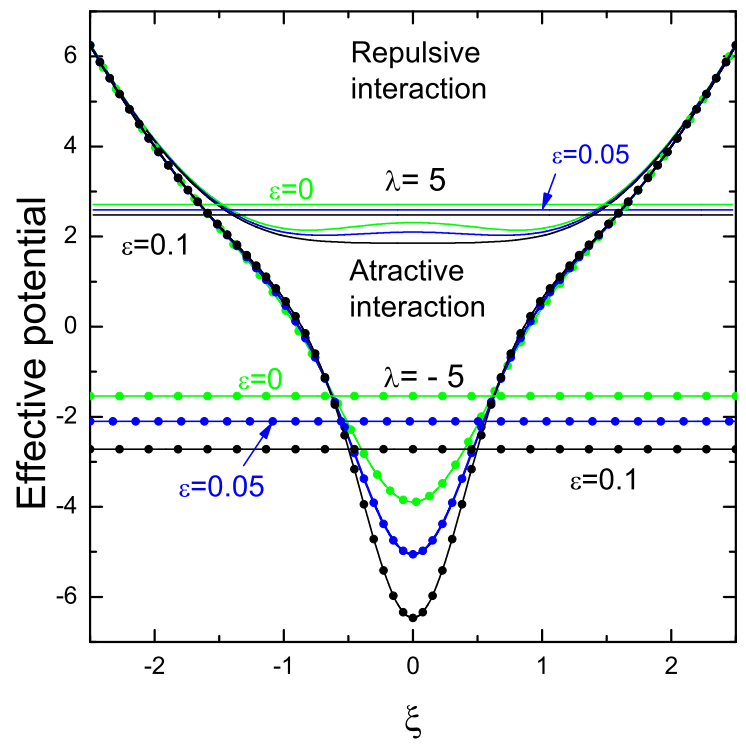

FIG. 3: (Color online) The potential $U_{\text {eff }}$ given by Eq. (28) for $\lambda=5$ (solid lines) and $\lambda=-5$ (dot-solid lines). In the calculation we employed the values of $\varepsilon=0$ (green), 0.05 (blue) and 0.1 (black). The chemical potential $\mu_{\varphi^{3}-\varphi^{5}}$ for each $\varepsilon$ is indicated by flat lines.

$$
U_{e f f}=\xi^{2}+\lambda\left|\varphi_{\lambda}\right|^{2}-\varepsilon \lambda^{2}\left|\varphi_{\lambda}\right|^{4}
$$

where the order parameter $\varphi_{\lambda}(\xi):=\psi_{\tau(\lambda)}(\xi)$ has been substituted by the trial function (12) with $\sigma_{\varphi^{3}-\varphi^{5}}(\lambda ; \varepsilon):=\sqrt{\tau_{\varphi^{3}-\varphi^{5}}}$. Figure (3) shows the potential $U_{\text {eff }}$ for both considered cases, attractive $(\lambda=-5)$ and repulsive interatomic $(\lambda=5)$ interactions. Also, in the figure is represented the values of $\mu_{\varphi^{3}-\varphi^{5}}$ for $\varepsilon=0$, 0.05 , and 0.1. It becomes clear that for a given $\lambda>0$, the variation of the function $U_{\text {eff }}(\xi ; \lambda, \varepsilon)$ with respect to the parameter $\varepsilon$ is negligible. Hence, the corresponding chemical potentials $\mu_{\varphi^{3}-\varphi^{5}}(\lambda, \varepsilon) \simeq \mu_{\varphi^{3}-\varphi^{5}}(\lambda, 0)$. Thus, the nonlinear potential $\lambda|\psi|^{2}$ for $\lambda>0$ becomes a very good description to study the physical properties of a 1D cigar-shape BEC under the condition that the transverse harmonic oscillator frequency $\omega_{\mathbf{r}}$ is much larger than the perpendicular frequency trap $\omega$. This result is in agreement with the conclusions of Ref. 12. As one can see from Fig. (2) the error $\Delta \mu_{\varphi^{3}-\varphi^{5}}(\lambda>0, \varepsilon)$ ranges between $2-7 \%$ with respect to the value of $\mu_{\varphi^{3}}(\lambda)$. Now, if we consider the opposite case, i.e. an attractive interaction, the behavior of $U_{\text {eff }}(\xi ; \lambda, \varepsilon)$ presents a strong localized potential and, in correspondence, the chemical potential will change drastically as the parameter $\varepsilon$ increases. This characteristic of the effective potential (28) for $\lambda<0$ determines clearly that the term $\lambda|\psi|^{2}$ is not enough for a correct description of $1 \mathrm{D}$ cigar-like shape BECs. In this case, the residual three dimensionality term $-\varepsilon \lambda^{2}|\psi|^{4}$, for the effective 1D GPE under a harmonic trap, strongly modify the corresponding chemical potential. Small variation of the strength $\varepsilon \lambda^{2}$ leads to strong change of $\mu_{\varphi^{3}-\varphi^{5}}$ value and hence the ground state energy $E_{\varphi^{3}-\varphi^{5}}$ as well.

This peculiar behavior is related to the orbital stability of the nonlinear Schrödinger equation (44). As mentioned above, the 3D GPE presents a set of ground states which is orbitally stable for any value of the self-repulsive interaction, while for the attractive interparticle interaction regime $\left(a_{s}<0\right)$ the solution does not collapse if and only if the condition $N a_{s}>-a_{\perp} 0.627$ is fulfilled. The former criterion can be rewritten as $-\lambda<\lambda_{3 D}=2.33 / \sqrt{\varepsilon}$ and it should be compared with condition of existence and validity, for $-\lambda<\lambda_{S}=2.857 / \sqrt{\varepsilon}$, of the obtained solutions Eqs. (22)-(27). In the inset Fig. (22) for the attractive interaction, the intersection of these two sets, $\lambda_{3 D}(\varepsilon) \cap \lambda_{S}(\varepsilon)$, is represented by a shaded region in the $\lambda-\varepsilon$ diagram.

In conclusion, a new variational approach is presented, which allows to construct for the cubic-quintic GPE closed analytical expressions for the order parameter, the minimal energy, and the corresponding chemical. Focusing on the compact analytical expressions, we report the contribution of the quintic term and the systematic error of the residual 3D contribution to the 1D cigar-shape model for both attractive and repulsive interaction. By the calculations the obtained chemical potential solution highlight the strong dependence on the sign of strength $\lambda$ and the values of quintic self-interaction parameter $\varepsilon$.

\section{Acknowledgments}

C.T-G acknowledges the hospitality at the MaxPlanck-Institut für Physik Komplexer Systeme and thanks Alexander von Humboldt Foundation for finan- 
cial support.

1 A. Görlitz, et al., Phys. Rev. Lett. 87, 130402, (2001); M. Greiner , I. Bloch, O. Mandel, T. W. Hänsch, and T. Esslinger, Phys. Rev. Lett. 87, 160405 (2001); F. S. Cataliotti, et al., Science 293, 843 (2001).

2 E. H. Lieb, Phys. Rev. 130, 1616 (1963); M. Olshanii, Phys. Rev. Lett. 81, 938 (1998); D. S. Petrov, G. V. Shlyapnikov and J. T. M. Walraven, Phys. Rev. Lett. 85, 3745 (2000).

3 E. H. Lieb, R. Seiringer, and J. Yngvason, Commun. Math. Phys. 244, 347 (2004).

4 R. Carretero-González, D. J. Frantzeskakis, and P. G. Kevrekidis, Nonlinearity 21, R139, (2008).

5 A. E. Muryshev, G. V. Shlyapnikov, W. Ertmer, K. Sengstock, and M. Lewenstein, Phys. Rev. Lett. 89, 110401 (2002).

6 L. Khaykovich and B. A. Malomed, Phys. Rev. A 74 , 023607 (2006).

7 Kh. I. Pushkarov, D. I. Pushkarov, and I. V. Tomov, Opt.
Quantum Electron., 11, 471 (1979).

8 S. Sinha, A. Y. Cherny, D. Kovrizhin, and J. Brand, Phys. Rev. Lett. 96, 030406 (2006); L. Salasnich, A. Parola , and L. Reatto, Phys. Rev. A 65, 043614 (2002).

9 V. M. Pérez-García, H. Michinel, and H. Herrero, Phys. Rev. A 57, 3837 1(998); A. Gammal, L. Tomio, and T. Frederico, Phys. Rev. A 66, 043619 (2002); L. D. Carr and Y. Castin, Phys. Rev. A 66, 0636022002.

10 R. Cipolatti, J. López Gondar, and C. Trallero-Giner, Physica D-Nonlinear phenomena 241, 755 (2012).

11 This type of equation, excluding the confinemet potential, have considered for studing the dynamic of the dark soliton and the properties of a 1D BEC [see V.A. Brazhnyi, V. V. Konotop and L. P. Pitaevskii, Phys. Rev. A 73 053601(2006) and reference therein].

12 E. H. Lieb, R. Seiringer, and J. Yngvason, Phys. Rev. Lett. 91, 150401 (2003). 